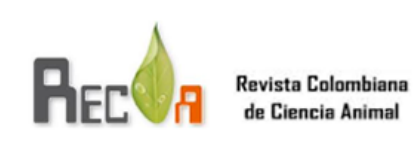

Revista Colombiana de Ciencia Animal ISSN: 2027-4297

reciaeditor@unisucre.edu

Universidad de Sucre

Colombia

\title{
Desempeño agronómico y valor nutritivo de Tithonia diversifolia (Hemsl.) A Gray para la alimentación de bovinos
}

Londoño C, Juan; Mahecha L, Liliana; Angulo A, Joaquín

Desempeño agronómico y valor nutritivo de Tithonia diversifolia (Hemsl.) A Gray para la alimentación de bovinos

Revista Colombiana de Ciencia Animal, vol. 11, núm. 1, 2019

Universidad de Sucre, Colombia

DOI: https://doi.org/10.24188/recia.v0.n0.2019.693

Los autores permiten a RECIA reimprimir el material publicado en él. En caso de que un autor quiera traducir o usar una publicación parcial o completa de nuestro Diario, el autor debe obtener un permiso por escrito del editor de la revista.

Esta obra está bajo una Licencia Creative Commons Atribución 4.0 Internacional. 


\title{
Desempeño agronómico y valor nutritivo de Tithonia diversifolia (Hemsl.) A Gray
} para la alimentación de bovinos

\author{
Agronomic potential and nutritional quality of Tithonia diversifolia (Hemsl.) A Gray for feeding cattle \\ Juan Londoño $C$ \\ DOI: https://doi.org/10.24188/recia.v0.n0.2019.693
}

SENA - CRNR La Salada, Caldas; Grupo de Investigación

La Salada; Antioquia., Colombia

kewns@misena.edu.co

Dttp://orcid.org/0000-0002-0688-7437

Recepción: 10 Octubre 2018

Aprobación: 27 Diciembre 2018

Liliana Mabecha L

Universidad de Antioquia, Facultad de Ciencias Agrarias;

Grupo de Investigación en Ciencias Agrarias (GRICA);

Medellin., Colombia

liliana.mahecha@udea.edu.co

iD http://orcid.org/0000-0003-3377-8399

Joaquin Angulo $A$

Universidad de Antioquia, Facultad de Ciencias Agrarias;

Grupo de Investigación en Ciencias Agrarias (GRICA);

Medellin, Colombia

joaquin.angulo@udea.edu.co

iD http://orcid.org/0000-0003-3352-8795

\section{RESUMEN:}

La producción ganadera tradicional debido a la presión ejercida sobre los recursos, debe sufrir una transformación que incluya elementos que permitan la sostenibilidad de la actividad en el entorno productivo. La implementación de sistemas silvopastoriles se constituye en una alternativa viable para el mejoramiento de la producción a través de la inclusión de especies leñosas en el potrero para la formación de diferentes estratos, que generen múltiples beneficios a todos los elementos del ciclo bovino. La selección de las especies silvopastoriles debe ser realizada teniendo en cuenta aspectos agronómicos y composicionales para optimizar la respuesta animal. Este documento presenta una revisión sobre el potencial agronómico, la calidad nutricional y el uso del botón de oro (Tithonia diversifolia - Asteraceae) en la alimentación de bovinos. Se describen las principales formas de establecimiento, contenido de nutrientes, producción de biomasa, acción en el suelo debido a su uso como abono verde y sus efectos productivos en la dieta de los rumiantes. El establecimiento del botón de oro (Tithonia diversifolia), puede ser realizado a través de semilla sexual y vegetativa. Su composición nutricional muestra niveles elevados de proteína y carbohidratos solubles, bajo contenido de FDN, y niveles adecuados de minerales, su establecimiento y uso como abono verde puede mejorar las condiciones de fertilidad del suelo para aumentar la producción y rendimiento del pasto. Estas características le confieren una alto potencial en la alimentación bovina, ya sea como recurso para ramoneo, procesado en harina o ensilado, para reducir los costos de producción en explotaciones ganaderas.

Palabras Clave: alimentación bovina, Asteraceae, métodos de siembra, producción de biomasa, sistemas silvopastoriles.

\section{ABstract:}

The traditional livestock, due to the pressure exerted on the resources, must undergo a transformation that includes elements that allow the sustainability of the activity in the productive environment. The implementation of silvopastoral systems constitute a viable alternative for the improvement of production through the inclusion of woody species in the pasture for the formation of different plant stratum, which brings multiple benefits to the bovine productive cycle. The selection of these species should be carried out taking into account agronomic and compositional aspects to optimize animal response. This paper presents a review of the Mexican Sunflower (Tithonia diversifolia - (Asteraceae), in feeding cattle. Main ways of establishment, nutrient content, biomass production, effect on the soil due to its use as a green manure and their productive effects in the diet of ruminants are described. Mexican Sunflower (Tithonia diversifolia) can be established by sexual or vegetative seed. Its nutritional composition 
shows high levels of protein and soluble carbohydrates, low neutral detergent fiber content (NDF), and balanced levels of minerals. The establishment of this specie and its use as green manure can improve soil fertility to increase production and yield of the pasture. These characteristics give it a high potential in bovine feeding, either as a resource for browsing, processed in flour or silage, to reduce production costs in livestock farms.

KEYWORDS: bovine feeding, Asteraceae, methods of planting, biomass production, silvopastoral system.

\section{INTRODUCCIÓN}

La ganadería en su enfoque tradicional propone el cambio del uso del suelo deforestando grandes extensiones de bosques para el establecimiento de pasturas, buscando la ampliación de la frontera agrícola para mantener los niveles productivos con fines comerciales o de subsistencia (1), lo que ha desencadenado una serie de efectos negativos sobre la base ecosistémica, afectando los recursos edáficos, hídricos y faunísticos (2). En Colombia aún imperan los sistemas de producción extensivos tradicionales, que se han caracterizado por el bajo rendimiento y por los efectos negativos causados en el ambiente, estos controlan casi la mitad de las praderas del país y cerca de dos terceras partes del inventario ganadero (3). A pesar del aumento en el área destinada a la producción de forrajes en los últimos 35 años en Colombia, pasando de 14,6 a 35,5 millones de ha (4), la productividad medida en términos de capacidad de carga es de 0.61 animales/ha (5), cifra desalentadora teniendo en cuenta el costo ambiental tan elevado que ha acarreado la implementación de sistemas de producción ganadera en el país.

Considerando estos bajos niveles de eficiencia, se crea la necesidad de realizar un cambio en el modelo productivo que propenda hacia el aumento en los rendimientos y que brinde al ganadero una opción de mejora de las actuales condiciones de la actividad. Los sistemas agroforestales ofrecen una alternativa para incrementar el nivel productivo de los bovinos, mitigar el impacto generado en el ambiente y reducir la dependencia de insumos externos (6,7). La inclusión de especies leñosas en asocio con pasturas en los potreros que serán la base sustentadora del alimento para los bovinos, requiere de una selección cuidadosa basada en las características de adaptación, desarrollo y valor nutricional.

Dentro del grupo de especies que se tiene como opción para diversificar la dieta de los bovinos se encuentra el Botón de oro (Tithonia diversifolia), que por su contenido nutricional y características agronómicas se consolida como una opción ideal para su utilización en sistemas silvopastoriles, en zonas que presenten las condiciones ideales para su desarrollo. Este documento presenta una revisión sobre el potencial agronómico y la calidad nutricional del Botón de oro (Tithonia diversifolia) en sistemas silvopastoriles.

Propagación y establecimiento del botón de oro. Tithonia diversifolia conocida vulgarmente como botón de oro, es un arbusto perteneciente a la familia de las asteráceas, originaria del sur de México y centro América desde donde se ha extendido a diferentes partes de mundo, se caracteriza por su alto grado de plasticidad ecológica, pudiéndose encontrar desde el nivel del mar hasta los $2500 \mathrm{msnm}$, con precipitaciones anuales entre 800 a $5000 \mathrm{~mm}$ y en diferentes tipos de suelo; tolera condiciones de acidez, de baja fertilidad y crece espontáneamente a orillas de caminos, ríos y carreteras (8). Su potencial forrajero está ligado directamente a su tolerancia a la poda y a su capacidad de rebrote, efecto que permite obtener gran cantidad de biomasa por unidad de área (9).

La propagación de esta planta se puede realizar a través de semilla sexual y semilla vegetativa, siendo este último el método más utilizado, aunque debido la pérdida de las estacas por la humedad en el suelo y el elevado volumen de material empleado en el establecimiento, se han venido desarrollando avances significativos en la propagación a través de semilla sexual con resultados sobresalientes (10).

La sección del tallo que se utilice para la propagación cuándo se establezca el cultivo a través de material vegetativo y la forma en que se ubique este en el suelo tiene una repercusión directa sobre la velocidad de crecimiento y la producción de biomasa. González et al (11), evaluaron el desempeño productivo de esta especie utilizando dos secciones del tallo (basal y media) y dos formas de ubicación de la estaca en el surco 
(acostada y parada), los cortes para la medición se realizaron durante un periodo de 2 años, en el periodo poco lluvioso cada 3 meses y en el periodo lluvioso cada 2 meses, el mejor desempeño en cuanto a crecimiento y producción de hojas se obtuvo al sembrar la parte media del tallo acostada en el surco con una profundidad de $15 \mathrm{~cm}$, estos resultados pueden deberse a la mayor superficie de contacto que tiene el tallo con el suelo al ubicarse la estaca de forma horizontal, lo que propicia un mayor desarrollo radicular y por ende una mayor probabilidad de captación de nutrientes, además la parte media del tallo suele poseer mayor cantidad de yemas que la zona basal, incrementando así las posibilidades de rebrote, promoviendo una mayor formación de hojas que en conjunto con las sustancias de reserva en esta zona del tallo aumentan la tasa fotosintética, así como la cantidad de auxinas exógenas que estimulan la diferenciación radicular (12). Lo anterior concuerda con un estudio similar realizado por Ruiz et al (13), donde se obtuvo un mayor rendimiento en cuanto a producción de biomasa, ubicando la estaca de forma horizontal en el suelo, aunque estos autores sugieren que no hubo diferencia en cuanto a la sección del tallo utilizada medio o basal. Esta información contrasta con lo encontrado por Salazar (1992), citado por Ríos (14), quién evaluó la eficiencia de desarrollo en la siembra 15 días después de ser realizada, encontrando un $94 \%$ de prendimiento en estacas tomadas de la parte más leñosa (basal), y $58 \%$ en las estacas procedentes de la parte media, encontrando también una menor proporción de raíces en plantas obtenidas en esta sección del tallo, característica que influye de forma directamente proporcional sobre factores productivos como la población de tallos y la cantidad de M.S por ha (11).

La información acerca de la propagación de esta especie por medio de semilla sexual es escasa, aunque la necesidad de desarrollar alternativas de propagación más efectivas ha fomentado la investigación en este campo y la presentación de resultados ha tenido un incremento significativo en los últimos años. Según Romero et al (15), se pueden obtener alrededor de 17.000 plántulas por cada $\mathrm{Kg}$ de semilla sexual de botón de oro preparando un germinador en un sustrato compuesto por suelo, arena y materia orgánica, protegiendo la semilla de la exposición solar directa y de la humedad excesiva, utilizando como fuente de semilla plantas adultas de más de 4 meses de edad.

Algunas semanas después el germinador sigue produciendo plántulas, pudiéndose deber esto al elevado grado de latencia que presenta la semilla, este puede ser reducido de forma significativa aplicando tratamientos pre-siembra tales como exposición a la luz, inmersión de las semillas en agua caliente o inclusive almacenando la semilla por un periodo de hasta 12 meses (16). El uso de este método de siembra es más viable en términos económicos, aunque el desarrollo inicial de la planta sea un poco más lento, el sistema radicular es más profundo lo que podría brindar un mejor anclaje y una mayor superficie para la captación de nutrientes (17). El porcentaje de pérdidas es significativamente menor con semilla sexual (entre el 5 y el $10 \%$ con semilla sexual contra 30 al 40\% con semilla vegetativa), y los costos de producción son menores reduciendo a la mitad los costos generados por la mano de obra.

La germinación de las semilla de Tithonia diversifolia está determinada por varios factores tales como la exposición a la luz, humedad, sustrato, pretratamiento, profundidad de siembra entre otros. Agboola et al (16), encontraron que la geminación inicial es relativamente baja, alrededor de $30 \%$ después de 5 días de la siembra, sin embargo al aplicar pretatamientos a la semilla como la inmerción de esta en agua caliente a una temperatura entre los 80 y los $100^{\circ} \mathrm{C}$, por un lapso de 30 a 60 segundos, se puede elevar la tasa de germinación a niveles entre 80 y $85 \%$, esto puede ser debido a que el aumento de la temperatura genera cambios metabólicos que finalizan el estado de latencia de la semilla, además permite el ingreso de agua y oxígeno y remueve sustacias que inhiben la germinación (18). Un porcentaje de geminación similar (81\%) fue alcanzado por Etejere et al (19), al realizar la siembra de semilla sexual en masetas. En este estudio se encontró que un factor que afecta de forma significativa el porcentaje de germinación, es la profundidad de siembra. Los autores evaluaron la respuesta de la germiación a 5 diferentes profundidades: 0, 0,5, 1,5, 2,5 y $3,5 \mathrm{~cm}$ respecto a la superficie del suelo, el mayor porcentaje de germinación se presentó en la profundidad 0 $\mathrm{cm}$, y disminuyó a medida que aumentaba la profundidad. En las profundidaes de 0 a $2,5 \mathrm{~cm}$ la emergencia se dio alredor de los 5 días, y en la profundidad de $3,5 \mathrm{~cm}$ la emergencia inició a partir del sexto día. 
TABLA 1

Efecto de la profundidad de siembra sobre el porcentaje de germiación

\begin{tabular}{cc}
\hline Profundidad de siembra (cm) & Porcentaje de germinación \\
\hline 0 & 76,8 \\
0,5 & 53,3 \\
1,5 & 53,3 \\
2,5 & 23,3 \\
3,5 & 5 \\
\hline
\end{tabular}

Fuente: Etejere et al (19)

El almacenamiento de las semillas aumenta de manera ostensible la tasa de germinación (19), esto al parecer es debido a que el embrión requiere de un elevado periodo de maduración para aumentar su viabilidad (20).

Etejere et al (19), reportan que el número de semillas por planta depende del número de flores allí encontradas; cada planta puede producir entre 35 y 212 flores, cada flor produce entre 32 y 52 semillas, según Gallego (17) el número de flores promedio por planta es de 88,7, y cada flor produce en promedio 61,2 semillas. Por cada planta de Tithonia diversifolia se pueden obtener entre 1120 y 13144 semillas, con un peso promedio por cada 1000 semillas de entre 6.42 y 7,5 g (19) valor cercano a lo encontrado por Gallego (17), quien reporta un peso de 7,36 g por cada 1000 semillas.

Valor nutricional y producción de biomasa a diferentes edades de cosecha del botón de oro (Tithonia diversifolia). Las especies leñosas seleccionadas para su inclusión en sistemas silvopastoriles, deben cumplir con características forrajeras que permitan la sostenibilidad alimenticia de los animales y que ayuden a alcanzar un balance nutricional adecuado de la dieta, logrando disminuir la dependencia de insumos externos que hacen menos eficiente en términos económicos la actividad ganadera.

La evaluación del comportamiento agronómico de estas especies en condiciones silvopastoriles de ramoneo, permite determinar el tiempo óptimo para su utilización, relacionado directamente con la oferta de biomasa, el aporte de nutrientes y un periodo de recuperación similar al de la especie rastrera, con el propósito de aprovechar al máximo todo su potencial productivo.

La capacidad forrajera de Tithonia diversifolia y su calidad nutricional, están determinados entre otros factores por el estado fenológico. Esta especie presenta una marcada resistencia a la poda, se recupera de forma rápida de cortes sucesivos y además posee una elevada tasa de rebrote, lo que le permite producir gran cantidad de biomasa (9).

Estudios científicos y observaciones hechas en campo muestran que los periodos de recuperación ideales para la especie bajo condiciones de corte coincide con el estado de prefloración, es decir, alrededor de los 50 días, esto con el fin de no causar deterioro al cultivo y asegurar una producción estable y duradera, sin embargo en sistemas silvopastoriles de ramoneo este periodo debe ser más prolongado (entre 60 y 90 días dependiendo de las condiciones agroclimáticas) (8).

En un estudio realizado por Lugo et al (9), donde se evaluó la producción de materia seca y proteína cruda de Tithonia diversifolia, a diferentes alturas $(20$ y $50 \mathrm{~cm})$ y edades de corte $(30,60$ y 85 días), se determinó que la altura de corte no incide sobre la producción de biomasa, caso contrario a la frecuencia de corte que tuvo un marcado efecto sobre la producción, presentándose el valor más elevado a los 85 días con un total de 2.58 Kg por planta, para corte a los 60 días $1,73 \mathrm{Kg}$ por planta y para corte a los 30 días $0,82 \mathrm{Kg}$ por planta, de igual manera Gualberto et al (21) evaluaron la producción de biomasa y la composición nutricional a 3 diferentes densidades de siembra $(0.75 \mathrm{~m}$ x $0.5 \mathrm{~m}, 0.75 \mathrm{~m}$ x $0.75 \mathrm{~m}, 1.00 \mathrm{~m}$ x $0.75 \mathrm{~m}$ ) y 3 frecuencias de cortes (prefloración, floración y pos-floración.) presentándose la mayor producción en la interacción de post-floración con una densidad siembra de $0.75 \mathrm{~m} \mathrm{x} 0.5 \mathrm{~m}(83,25 \mathrm{t} / \mathrm{ha})$

Según Ríos (14), Tithonia diversifolia presenta una producción potencial de 37,92 t/ha, de material consumible (hojas, peciolos y tallos hasta de 2,0 cm de diámetro) con una densidad de siembra de 2,66 
plantas $/ \mathrm{m}^{2}$, realizando cortes cada 7 semanas. En cuanto la producción de materia seca, Castillo et al (22) obtuvieron a una densidad de siembra de $1 \mathrm{~m}$ x $1 \mathrm{~m}$ un total de $13,52 \mathrm{t} /$ ha en cortes realizados entre los 90 y los 100 días.

A pesar de no pertenecer a la familia de las leguminosas, el forraje de Tithonia diversifolia ha sido distinguido por aportar una cantidad significativa de proteína, en un estudio realizado por Calsavara et al (23), quienes evaluaron la producción de biomasa y el contenido nutricional de Tithonia diversifolia en 2 etapas de maduración inicio y prefloración, obtuvieron niveles de proteína de $16,5 \%$ y 14,9\%, en la planta entera, y para las hojas de 22,5\% y 22,3\% respectivamente, mientras que Gallego et al (10), encontraron niveles de $14,1 \%$ de proteína para plantas completas obtenidas a partir de semilla vegetativa a una edad de 56 días.

Lezcano et al (24) realizaron una caracterización bromatológica de la especie en 2 estados fisiológicos (30 y 60 días) en el periodo seco y lluvioso, obteniendo niveles de proteína para hojas de 29,79\% a los 30 días y de $17,27 \%$ a los 60 días para la época lluviosa. La degradabilidad en rumen de la proteína aportada por esta especie oscila entre $72,63 \%$ y $76,94 \%$ teniendo en cuenta que no se ve afectada por la cantidad de metabolitos secundarios (25), valor cercano a lo encontrado por Vargas (26), quien reporta una degradabilidad del 79\%. Esta depende de varios factores inherentes básicamente a la fisiología del animal y a las características de la proteína, aunque se considera la solubilidad como uno de los principales determinantes, pues a medida que aumenta está en el líquido ruminal, aumenta también la proteólisis (27). La variabilidad de los contenidos de proteína descritos en los estudios mencionados se debe básicamente a la parte de la planta analizada (hojas, tallos o planta completa), al estado fenológico, y a las condiciones agroclimáticas y de fertilidad del suelo.

El contenido de fibra detergente neutra se encuentran alrededor de 47,5\% en la planta entera para el estado de maduración (23), valor que está por debajo de lo descrito por Gallego et al (10) quienes reportan que la planta entera posee un nivel de 53,81\% de fibra detergente neutra a una edad de 56 días. En el cuadro 2 se describe el contenido nutricional de Tithonia diversifolia según lo reportado por diferentes autores.

En cuanto al contenido de minerales, esta especie posee una cantidad apreciable de fósforo alcanzando niveles entre $0,17 \%$ y $0,38 \%$ en las hojas, estos valores son más altos aún que los encontrados en algunas leguminosas usadas en sistemas agroforestales que varían entre 0,15 y $0,20 \%$ de fósforo $(28,29)$, el contendido de calcio se encuentra alrededor de $2,86 \%$ en la planta completa a los 56 días (10). 
TABLA 2

Contenido nutricional de Tithonia diversifolia

\begin{tabular}{|c|c|c|c|}
\hline Nutriente & Cantidad (\%) & D.S & Autores \\
\hline \multirow{7}{*}{ MS } & 9,35 & 1,10 & Lezcano et al. (24) \\
\hline & 22,40 & 2,50 & De Souza (30) \\
\hline & 25,26 & 4,97 & Verdecia et al. (31) \\
\hline & 17,91 & 3,32 & Navarro et al. (32) \\
\hline & 12,74 & & Gallego et al. (10) \\
\hline & 21,16 & 1,61 & Calsavara et al. (23) \\
\hline & 18 & $*$ & Cardona (33) \\
\hline \multirow{7}{*}{ PB } & 22,05 & 0,17 & Lezcano et al. (24) \\
\hline & 12,16 & 1,76 & De Souza (30) \\
\hline & 24,52 & 5,40 & Verdecia et al. (31) \\
\hline & 22,61 & 5,59 & Navarro et al. (32) \\
\hline & 14,01 & & Gallego et al. (10) \\
\hline & 15,65 & 1,06 & Calsavara et al. (23) \\
\hline & 18,3 & & Cardona (33) \\
\hline \multirow{5}{*}{ FDN } & 64,75 & 1,04 & De Souza (30) \\
\hline & 47,00 & 3,43 & Verdecia et al. (31) \\
\hline & 53,81 & & Gallego et al. (10) \\
\hline & 49,75 & 3,18 & Calsavara et al. (23) \\
\hline & 44,3 & & Cardona (33) \\
\hline \multirow{5}{*}{ FDA } & 42,53 & 2,97 & De Souza (30) \\
\hline & 29,17 & 2,55 & Verdecia et al. (31) \\
\hline & 48,18 & $*$ & Gallego et al. (10) \\
\hline & 34,34 & 2,93 & Calsavara et al. (23) \\
\hline & 33,3 & & Cardona (33) \\
\hline \multirow{3}{*}{ Lignina } & 7,90 & 0,67 & De Souza (30) \\
\hline & 22,81 & 14,08 & Verdecia et al. (31) \\
\hline & 15,58 & 3,05 & Calsavara et al. (23) \\
\hline \multirow{4}{*}{$\mathrm{Ca}$} & 2,02 & 0,04 & Lezcano et al. (24) \\
\hline & 1,55 & 0,26 & De Souza (30) \\
\hline & 2,86 & * & Gallego et al. (10) \\
\hline & 0,9 & * & Cardona (33) \\
\hline \multirow{4}{*}{$\mathrm{P}$} & 0,31 & 0,12 & De Souza $(30)$ \\
\hline & 0,35 & 0,02 & Navarro et al. (31) \\
\hline & 0,27 & * & Gallego et al. (10) \\
\hline & 0,4 & $*$ & Cardona (33) \\
\hline \multirow{4}{*}{ Cenizas } & 12,99 & 0,47 & Lezcano et al. (24) \\
\hline & 13,11 & 2,47 & Navarro et al. (32) \\
\hline & 16,19 & * & Gallego et al. (10) \\
\hline & 15 & $*$ & Cardona (33) \\
\hline
\end{tabular}

Fuente: Compilado por los autores.

Efecto del botón de oro (Tithonia diversifolia) en el suelo. El suelo comprendido como un cuerpo natural, dinámico, vivo y que se modifica constantemente, es el encargado de dar sostén y nutrir a la planta de la cual el animal obtendrá todos los nutrientes requeridos para su mantenimiento y producción. Desde la visión holística, el suelo determina casi todas las condiciones económico-sociales para el desarrollo de las poblaciones, pero debido a algunas prácticas inadecuadas en la implementación de sistemas ganaderos, se ha generado en este un estado de deterioro, resultando en una disminución de su capacidad sustentadora, lo que ha promovido el uso de fertilizantes de síntesis química que aportan solo una pequeña cantidad de los elementos que necesita la planta (34), por lo que ha surgido la necesidad de utilizar fuentes orgánicas de nutrientes que mejoren la fertilidad de suelo y que no representen un sobrecosto.

Diferentes autores reportan que existen especies arbóreas y arbustivas que además de ser fuente de alimento para animales, pueden ser utilizadas como combustible, y material de construcción (35), biocontroladores de plantas e insectos $(36,37)$, fitorremediadores de suelos $(38,39)$, coadyuvantes en la detención de la erosión, mejoradores de la fertilidad y rendimiento de cultivos como maíz (40), arroz (41), yuca (42), pasto elefante 
(P. purpureum) (43), encontrándose dentro de este grupo Tithonia diversifolia. Esta especie posee una cantidad apreciable de nutrientes y produce abundante materia seca por unidad de área, la cual se descompone rápidamente en el suelo convirtiéndose en una fuente de nutrientes para las plantas (44), aumentado de forma significativa el rendimiento y la calidad de los cultivos. En un estudio realizado por Sao et al (45), se evaluó la utilidad de Tithonia diversifolia en el incremento de la producción de biomasa y el efecto en la fertilidad del suelo en 3 tratamientos: 1) cultivo de Tithonia diversifolia, 2) cultivo de Tithonia diversifolia + pasto guinea, 3) cultivo de pasto guinea. Se encontró que la fertilidad del suelo medida a través del método de Bio-test (Phan Gia Tan y Preston 1995), fue superior en el tratamiento 2, presentándose una mayor producción de biomasa para el maíz cultivado en el suelo tomado de las parcelas plantadas únicamente con Tithonia diversifolia y mejorando la composición química del suelo 6 meses después del establecimiento. En otros estudios realizados por Gachengo et al (46), se demostró que al incorporar biomasa fresca de Tithonia diversifolia en una cantidad equivalente a 5 ton/ha de M.S en conjunto con una fuente inorgánica de $\mathrm{P}$ (Superfosfato triple) se aumentó el rendimiento de un cultivo de maíz medido a través de la producción de grano en 3 cosechas, de 2,7 ton/ ha en el tratamiento testigo donde no se utilizó ningún tipo de producto a 8,6 ton/ha. Este efecto también se ha observado en cultivos como el de pasto de $P$. purpureum vc. Cuba CT-169, según Crespo et al (43), al adicionar materia verde fraccionada en cantidades de 12 y 24 ton/ha de Tithonia diversifolia al suelo, se aumenta de manera significativa el número de plantones $/ \mathrm{m}^{2}$, hijos/planta y producción de materia seca/ha, además se mejoraron algunas características del suelo como densidad aparente, contenido de materia orgánica y $\mathrm{N}$ total. Resultados similares reportaron Ojeniyi et al (40) quienes evaluaron el efecto de Tithonia diversifolia en las propiedades fisicoquímicas del suelo, encontrando que suelos cultivados con esta especie presentaron menor densidad aparente, mayor porosidad y materia orgánica, comparado con suelos en los que se han establecido otro tipo de cultivos.

El efecto positivo generado por Tithonia diversifolia, en el rendimiento de los cultivos y en algunas características del suelo puede deberse a que uno de los principales limitantes para el desarrollo eficiente de las plantas es la baja disponibilidad del P, lo que es característico en la mayor proporción de los suelos tropicales, además una parte importante de este elemento adicionado a través de los fertilizantes químicos, es adsorbido por los óxidos de hierro y aluminio de la arcilla mineral, disminuyendo la cantidad disponible para la plantas (46). La utilización de abono orgánico aumenta disponibilidad biológica del $\mathrm{P}$, al aumentar: las cantidades aportadas de $\mathrm{P}$ orgánico e inorgánico, el suministro de $\mathrm{P}$ a la raíz facilitando el transporte difusivo lo que está ligado a la mejora en las propiedades físicas del suelo y a la reducción de la capacidad de adsorción, debido a la inactivación de los sitios de adsorción de $\mathrm{P}$ por productos de la descomposición de la materia orgánica $(47,48)$, adicionalmente el corto ciclo de esta planta permite incrementar la transferencia de biomasa aumentado el aporte de este y otros elementos. Otra razón puede ser que debido a la capacidad de esta especie para acondicionar el suelo a través de su sistema radicular, genera el ambiente adecuado para el establecimiento y proliferación de hongos que aumentan la biodisponibilidad del P. (40). Otro aspecto a resaltar, es el contenido de $\mathrm{N}$ en esta planta, el cual es aportado al suelo en cantidades suficientes para el favorecimiento del desarrollo de las plantas (43).

Los beneficios aportados por Tithonia diversifolia, no se reflejan únicamente en el efecto generado sobre la fertilidad del suelo. Una característica que tiene alta relevancia en los sistemas de producción para la conservación de los recursos hídricos, faunísticos y edáficos, es su potencial como planta acumuladora de metales pesados, estos elementos tóxicos representan un grave problema debido a los efectos adversos generados en la salud de humanos y animales y en el ambiente, ya que son altamente persistentes y poseen un alto grado de bioacumulación. Estos no son degradados fácilmente en el suelo, por lo que requieren ser removidos (49), lo que puede realizarse a través de procedimientos químicos, físicos y biológicos; las técnicas físicas y químicas son costosas y además afectan de forma irreversible las propiedades del suelo y la biodiversidad, puede hacer el suelo inservible para el crecimiento de las plantas, y representan una alta inversión. Por el contrario las técnicas biológicas suelen ser menos costosas y remueven los contaminantes 
sin afectar la fertilidad del suelo (50). Una de las principales características que debe tener una planta para ser utilizada en procesos de fitorremediación es su producción elevada de biomasa y la capacidad de concentrar grandes cantidad de metales en las parte aéreas (49), particularidades propias de Tithonia diversifolia. Adesodun et al (39) evaluaron el potencial de esta especie como fitorremediador en la extracción de elementos como $\mathrm{Pb}$ y $\mathrm{Zn}$, utilizando suelo llevado artificialmente a la saturación máxima crítica (400 $\mathrm{mg} / \mathrm{kg}$ ), realizando mediciones de la concentración de estos elementos en la parte aérea de la planta (hojas y tallos), en la raíz y el remanente en el suelo cada 4, 6 y 8 semanas después de la siembra, encontrando que Tithonia diversifolia fue capaz de extraer más de la mitad de la concentración inicial de $\mathrm{Pb}$ y acumular grandes cantidades de Zn. En este estudio también se evidenció que la mayor proporción estos elementos se concentraron en la raíz seguido de las hojas y por último en el tallo, teniendo en cuenta que los contenidos de $\mathrm{Pb}$ y $\mathrm{Zn}$ disminuían conforme avanzaban los periodos de medición.

Uso del de botón de oro (Tithonia diversifolia) en la alimentación bovina. En Colombia la producción ganadera se desarrolla en zonas donde las gramíneas tropicales de bajo valor nutricional son la principal fuente de alimento, estas como único componente de la dieta no ofrecen los nutrientes necesarios para suplir los requerimientos de los animales, por lo que actualmente se buscan materiales para utilizar en la dieta como suplemento, que aporten alto contenido de proteína, carbohidratos solubles y que mejoren el balance ruminal representando menores costos energéticos por la producción de metano, mitigando el impacto ambiental y aumentando la calidad de la leche (17). Tithonia diversifolia posee características óptimas para ser incluida en la producción animal, y ha sido utilizada en la alimentación de cerdos, aves, conejos, peces, bovinos, entre otros (51) La utilización de esta planta para ramoneo, no ha sido descrita ampliamente en artículos técnicos, generalmente lo reportado es su comportamiento en sistemas de corte y acarreo (52), no obstante existen trabajos como los realizados por Rivera et al (53), quienes evaluaron el desempeño de vacas cruzadas (Bos taurus x Bos indicus) en el Piedemonte Amazónico, en un sistema silvopastoril intensivo (SSPi) con Tithonia diversifolia y en un monocultivo, encontrando que la producción de leche fue mayor en un 7\% en el SSPi. En cuanto al contenido de sólidos totales (ST), no se encontraron diferencias entre los tratamientos por animal, pero si se detectó un incremento en la producción de ST/ha del 36,2\% en el SSPi. Igualmente, en una investigación realizada en trópico de altura con vacas Holstein en producción, pastoreando en un SSP de kikuyo (Cenchrus clandestinus) y Tithonia diversifolia y en un monocultivo de kikuyo (Cenchrus clandestinus), se obtuvieron incrementos en el volumen y en la proteína en favor de los animales en el SSP (Monocultivo: 24,89 1/vaca/día, y Proteína 2,89 \%. SSP: 28,24 l/vaca/día, y Proteína $3 \%$ ), adicionalmente se observó una marcada diferencia en el recuento de células somáticas presentando en el SSP 67.26 x1000/ $\mathrm{ml}$, y en el monocultivo $125.35 \times 1000 / \mathrm{ml}$ (33), hecho que puede ser atribuido a la presencia de lactonas sesquiterpénicas en Tithonia diversifolia, compuestos que actúan en la inhibición de la activación del factor de transcripción NF- $\kappa \mathrm{B}$, disminuyendo así la síntesis de mediadores inflamatorios tales como citoquinas y quimioquinas (54). En otro estudio, Mahecha et al (55), efectuaron un reemplazo del $35 \%$ del alimento concentrado comercial en la alimentación de vacas F1 (Holstein x Cebú), sin tener cambios en el volumen producido y en la calidad composicional de la leche. De igual manera, en un ensayo realizado por Gallego (17), donde se analizó el efecto de 3 niveles diferentes de inclusión de harina de botón de oro (0, 15\% y 25\%) en el suplemento alimenticio de vacas Holstein, no se obtuvieron diferencias significativas en la producción y calidad composicional de la leche entre los tratamientos, por lo que se puede concluir que el botón de oro es una excelente alternativa para la suplementación de vacas lecheras ya sea bajo pastoreo directo o en harina.

El efecto favorable observado en la calidad composicional de la leche puede estar relacionado con la presencia moderada de metabolitos secundarios contenidos en esta especie, específicamente con los niveles de taninos, debido a la acción de estos en la producción de proteína sobrepasante, al formar complejos con la proteína endógena, lo que favorece la digestión postruminal, contribuyendo de esta manera a mejorar la respuesta productiva de los bovinos (56). 
Además esta especie aporta una alta cantidad de carbohidratos solubles, que juegan un papel muy importante en la fermentación a nivel ruminal para la formación de Ácidos Grasos Volátiles, favoreciendo la producción de leche.

En Colombia la disponibilidad de forraje para la alimentación de los bovinos en época seca es limitada, lo cual requiere del desarrollo de tecnologías que permitan afrontar el desabastecimiento de alimento, además que aporten una elevada cantidad de nutrientes y que no representen un sobrecosto. La utilización de la técnica del ensilaje permite el almacenamiento de los excedentes de forraje obtenidos en la temporada de lluvias sin alterar de manera significativa la composición nutricional y la palatabilidad del material conservado. El ensilaje ofrece ventajas ante otros métodos de conservación como por ejemplo a través de la técnica de henificación, debido a que es menos dependiente de las condiciones climáticas y permite conservar mayor cantidad de especies (57). Diferentes autores han señalado que el botón de oro puede ser conservado a través de esta técnica, mezclado en proporciones diferentes con otros recursos forrajeros como complemento que aporten una cantidad significativa de carbohidratos solubles, como sustrato de las bacterias acidolácticas, para el favorecimiento de la fermentación. Dávila et al (58) evaluaron la utilidad nutricional del ensilaje de Sorgo (Sorghum vulgare) y Botón de oro (Tithonia diversifolia) en tres tratamientos con proporciones diferentes, (T1: 75/25, T2: 50/50, y T3: 25/75. Sorgo/Botón), obteniendo que la mezcla con las proporciones $75 / 25$ Sorgo/Botón, puede ser considerada la mejor opción para la inclusión en la dieta de los animales, teniendo en cuanta el contenido de: M.S: T1: 30\%. T2: 27.10\% y T3: 25.87\%, ELN: T1: 44.49\%, T2: 42.89\% y T3: 42.51\% TDN T1: 34.45\%. T2: 21.19\% y T3: 21.02\%, y su calificación sensorial determinada por la metodología de consulta a expertos. Cabe anotar que a medida que aumentó la proporción de Tithonia diversifolia en la mezcla, aumentó de forma significativa la cantidad de proteína y de cenizas, y disminuyó la cantidad de fibra cruda. En un estudio similar, Gutiérrez et al. (59) evaluaron el efecto del ensilaje de la mezcla de Tithonia diversifolia con pasto Pennisetum purpureum vc. Cuba CT-169 en proporciones de 20:80, 40:60, 60:40 y 80:20 \% Botón/Pasto, e inoculada con el producto biológico comercial (VITAFERT) en cantidades de 0; 4.5; 6.0; $8.0 \%$. Los mejores resultados en cuanto a PB, concentración de ceniza y reducción de FND, se obtuvieron al adicionar cantidades entre 4,5 y $6 \%$ del inóculo comercial.

Los inoculantes tienen la capacidad de incrementar el rendimiento animal a través del consumo de ensilaje hasta en un $7 \%$, esto es debido al incremento de la digestibilidad del material, en lugar del aumento del consumo. Las bacterias acidolácticas no son celulolíticas por ende el efecto del incremento de la digestibilidad puede ser debido básicamente a la fermentación extensiva del sustrato a ácido láctico en los ensilajes, bajo estas condiciones habrá hidrólisis de los carbohidratos estructurales a azúcares solubles. Se ha demostrado igualmente que los ensilajes altamente fermentados son consumidos de forma más lenta por los animales, este cambio en el comportamiento ingestivo influencia el tiempo de retención ruminal, lo que puede también explicar el aumento en la digestibilidad de los ensilajes inoculados (57). El contenido de proteína y carbohidratos fermentables en ensilajes mixtos de gramíneas con arbustivas tiende a aumentar a medida que se incremente la proporción de la arbustiva (60).

A pesar de ser una especie promisoria por su potencial productor y su contenido nutricional, Tithonia diversifolia posee algunas limitaciones principalmente en su desempeño agronómico, que brindando algunas condiciones de manejo pueden ser controladas y que no suponen un impedimento para destacar las bondades de esta especie. A la hora de realizar el establecimiento de las plantas de Tithonia diversifolia, se debe tener en cuenta: seleccionar un terreno que cuente con buen drenaje y permeabilidad, control de aguas subterráneas y distancia de fuentes hídricas, pues esta planta presenta una tolerancia baja a zonas con encharcamiento, igualmente se debe tener en cuenta no establecer el cultivo en zonas donde se pueda presentar sombrío excesivo, que evite la intercepción de la energía luminosa por parte de esta especie, debido su baja tolerancia al sombrío (61). 
Agradecimientos: Los autores agradecen al Proyecto CODI 2014-910, de la Universidad de Antioquia y al SENA Centro de los Recursos Naturales Renovables La Salada, por el apoyo económico brindado para la ejecución de este trabajo.

\section{ReFERENCIAS}

1. Jadán $\mathrm{O}$, Cedillo $\mathrm{H}$, Zea $\mathrm{P}$, Quichimbo $\mathrm{P}$, Peralta Á, Vaca C. Relación entre deforestación y variables topográficas en un contexto agrícola ganadero, cantón Cuenca. Bosques Latitud Cero. 2017; 6(1):1-13. http:// revistas.unl.edu.ec/index.php/bosques/article/view/179

2. Mora Marín M, Ríos Pescador L, Ríos Ramos L, Almario Charry J. Impacto de la actividad ganadera sobre el suelo en Colombia. Ingeniería Y Región, 2017; 1-12. https://doi.org/10.25054/22161325.1212

3. Estrada J. Pastos y forrajes para el trópico colombiano. Universidad de Caldas: Caldas Colombia; 2002.

4. Instituto Alexander Von Humboldt. Colombia biodiversidad siglo XXI. Santafé de Bogotá, Colombia; 1998.

5. DANE. Boletín técnico comunicación informativa, Encuesta Nacional Agropecuaria ENA- 2016. Bogotá, Colombia: Dane; 2016. URL Disponible en: https://www.dane.gov.co/files/investigaciones/agropecuario/ enda/ena/2016/boletin_ena_2016.pdf

6. Carvajal T, Lamela L, Cuesta A. Evaluación de las arbóreas Sambucus nigra y Acacia decurrens como suplemento para vacas lecheras en la Sabana de Bogotá Colombia. Pastos y Forrajes. 2012; 35(4):417-430. http:// scielo.sld.cu/scielo.php?script=sci_arttext\&pid=S0864-03942012000400007\&lng=es\&tlng=es

7. Soto L, Jiménez G. Contradicciones socioambientales en los procesos de mitigación, asociados al ciclo del carbono en sistemas agroforestales. Madera y Bosques. 2018; 24. DOI: https://doi.org/10.21829/myb.2018.2401887

8. Calle Z, Murgueitio E. El Botón de Oro: Arbusto de Gran Utilidad Para Sistemas Ganaderos de Tierra Caliente y de Montaña. Revista Carta Fedegán. 2008; 108:54-63. http://www.fedegan.org.co/carta-fedegan-108alimentacion-y-productividad

9. Lugo M, Molina F, Gonzáles I, Gonzáles J, Sanchéz E. Efecto de la altura y frecuencia de corte sobre la producción de materia seca y proteína bruta de Tithonia diversifolia. Zootecnia Tropical. 2012; 30(4):317-325.

10. Gallego L, Mahecha L, Angulo J. Calidad Nutricional de Tithonia diversifolia Hemsl. A Gray bajo tres sistemas de siembra en el trópico alto. Agronomía Mesoamericana. 2017; 28(1):213-222. https://doi.org/10.15517/ am.v28i1.22673

11. González D, Ruiz E, Díaz H. Sección del tallo y forma de plantación: su efecto en la producción de biomasa de Tithonia diversifolia. Revista Cubana de Ciencia Agrícola. 2013; 47(4):425-429, 2013. http://www.redalyc.org/ pdf/1930/193029815017.pdf

12. Fanego A, Soto R, Martínez S. Brotación y Enraizamiento de Estacas de diferentes secciones de las ramas de Bougainvillea glabra choisy. Centro Agrícola 2009; 36(3):9-13. http://cagricola.uclv.edu.cu/index.php/es/volumen-36-2009/numero-3-2009/446-brotacion-yenraizamiento-de-estacas-procedentes-de-diferentes-secciones-de-las-ramas-de-bougainvillea-glabra-choisy

13. Ruiz E, Febles G, Díaz H, Achang G. Efecto de la sección y el método de plantación del tallo en el establecimiento de Tithonia diversifolia. Revista Cubana de Ciencia Agrícola. 2009; 43(1):91-94.

14. Ríos KC. Tithonia Diversifolia ( Hemsl .) Gray, una planta con potencial para la producción sostenible en el trópico. FAO. Agroforestería para la producción animal en latinoamérica. [En linea] 1998. URL Disponible en:

15. Romero O, Galindo A, Murgueitio E, Calle Z. Primeras experiencias en la propagación del botón de oro (Tithonia diversifolia, Hemsl. Gray) a partir de semillas para la siembra de sistemas silvopastoriles intensivos en Colombia. Tropical and Subtropical Agroecosystems. 2014; 17(3):525-528

16. Agboola A, Idowu F, Kadiri M. Seed Germination and Seedling Growth of the Mexican Sunflower Tithonia Diversifolia (Compositae) in Nigeria, Africa. Revista de Biología Tropical. 2006; 54(2):395-402. 
17. Gallego L. Evaluación agronómica y análisis productivo del botón de oro (Tithonia diversifolia (Hemsl. A Gray) como suplemento alimenticio de vacas lecheras en trópico alto. (Tesis de Maestría). Universidad de Antioquia: Medellín: 2016. http://bibliotecadigital.udea.edu.co/handle/10495/6113

18. Nikolaeva M. Physiology of Deep Dormancy in Seeds (Fiziologiya Glubokogo Pokoya Semyan). Israel Program for Scientific Translations [available from the U.S. Dept. of Commerce, Clearinghouse for Federal Scientific and Technical Information, Springfield, Va.]. 1969.

19. Etejere E. Olayinka U. Seed production, germination, emergence and growth of Tithonia diversifolia ( HEMSL ) A Gray as influenced by different sowing depths and soil types. American-Eurasian J Agric \& Environ Sci. 2014; 14(5):440-444. . https://www.idosi.org/aejaes/jaes14(5)14/9.pdf

20. Li X, Baskin M, Baskin C. Physiological Dormancy and Germination Requirements of Seeds of Several North American Rhus Species (Anacardiaceae). Seed Science Research. 1999; 9(3):237-245. https://doi.org/10.1017/ S0960258599000240

21. Gualberto R, De Sousa O, Costa N, Braccialli C, Gaion L. Influência do espaçamento e do estádio de desenvolvimento da planta na produção de biomassa e valor nutricional de Tithonia diversifolia (hemsl.) Gray. Nucleus. 2011; 8(1):241-255. http://dx.doi.org/10.3738/nucleus.v8i1.428

22. Castillo R, Betancourt T, Toral O, Iglesias J. Influencia de Diferentes Marcos de Plantación en el Establecimiento y La Producción de Tithonia diversifolia. Pastos y Forrajes. 2016; 39(2):89-93. http://scielo.sld.cu/pdf/pyf/ v39n2/pyf02216.pdf

23. Calsavara F, Ribeiro R, Silveira S, Delarota G, Freitas D, Sacramento J, et al. Productivity and chemical characteristics of Tithonia diversifolia forage. Argentina; $3^{\circ}$ Congreso nacional de sistemas silvopastoriles : vii congreso internacional sistemas agroforestales. Ediciones INTA; 2015. URL Disponible en: http://inta.gob.ar/ sites/default/files/script-tmp-libro_actas_silvopastoriles_-_agroforestales.pdf

24. Lezcano Y, Soca M, Ojeda F, Roque E, Fontes D, Montejo L, et al. Caracterización bromatológica de Tithonia diversifolia (HEMSL) A Gray, en dos etapas de dos etapas de su ciclo fisiológico. Pastos y forrajes. 2012; 35(3):275-282. http://scielo.sld.cu/pdf/pyf/v35n3/pyf03312.pdf

25. Medina M, García D, González M, Cova L, Moratinos P. Variables morfo-estructurales y de calidad de la biomasa de Tithonia diversifolia en la etapa inicial de crecimiento. Zootecnia Tropical. 2009; 27(2):121-134. http:// www.scielo.org.ve/pdf/zt/v27n2/art03.pdf

26. Vargas E. Caracterización de recursos forrajeros disponibles en tres agroecosistemas del Valle del Cauca. Cali, Colombia: Memorias II Seminario Internacional Desarrollo sostenible de Sistemas Agrarios; 1994. URL Disponible en: http://documentacion.ideam.gov.co/cgi-bin/koha/opac-detail.pl?biblionumber=2018

27. Mendoza R, Mohar F. Metabolismo animal, Volumen 1, Ediciones EDIUDCA Universidad de Ciencias Aplicadas y Ambientales UDCA: Bogotá Colombia; 2001

28. Palm A. Contribution of agroforestry trees to nutrient requirements of intercropped plants. Agroforestry Systems. 1995; 30(1-2):105-124. http://dx.doi.org/10.1007/978-94-017-0681-0_5

29. Wanjau S, Mukalama J, Hijssen R. Biomass Transfer: Harvesting Free Fertiliser.ILEIA Newsletter. 1994; $13(3): 25-28$.

30. De Souza O. Influência do espaçamento e do estádio de desenvolvimento da planta na produção de biomassa e valor nutricional de Tithonia diversifolia (HEMSL.) Gray (Tesis de Maestría). Universidad de Marília, Faculdad de Ciencias Agrarias: Brasil; 2007.

31. Verdecia D, Ramírez L, Leonard Y, Bazán Y, Bodas R, et al. Calidad de la Tithonia diversifolia en una zona del Valle del Cauto. REDVET. Revista Electrónica de Veterinaria. 2011; 12(5):1-13

32. Navarro F, Rodríguez EF. Estudio de algunos aspectos bromatológicos del Mirasol (Tithonia diversifolia Hemsl y Gray) como posible alternativa de alimentación animal. (Doctoral dissertation) Universidad del Tolima: Ibagué, Colombia; 1990.

33. Cardona L. Efecto de la mezcla de Tithonia diversifolia - Cenchrus clandestinus y la adición de ácidos grasos polinsaturados sobre la producción de metano in vitro y la medición in vivo de del balance nitrogenado y el desempeño productivo de vacas Holstein (Tesis de Maestría). Universidad de Antioquia; Medellín: 2017. 
34. Primavesi A, Molina J. Manejo ecológico del suelo: la agricultura en regiones tropicales, 5 edición ilustrada, Buenos Aires, Argentina: El Ateneo; 1984.

35. Olabode OS, Sola O, Akanbi WB, Adesina GO, Babajide PA. Evaluation of Tithonia diversifolia (Hemsl.) A Gray for soil improvement. World Journal of Agricultural Sciences. 2007; 3(4):503-7.

36. Tongma S, Kobayashi K, Usui K. Allelopathic activity of Mexican sunflower [Tithonia diversifolia (Hemsl.) A. Gray] in soil under natural field conditions and different moisture conditions. Weed Biology and Management. 2001; 1(2):115-9. https://doi.org/10.1046/j.1445-6664.2001.00020.x.

37. Bagnarello G, Hilje L, Bagnarello V, Cartín V, Calvo M. Actividad fagodisuasiva de las plantas Tithonia diversifolia y Montanoa hibiscifolia (Asteraceae) sobre adultos del insecto plaga Bemisia tabaci (Homoptera: Aleyrodidae). Rev Biol Trop. 2009; 57(4):1201-1215. https://doi.org/10.15517/rbt.v57i4.5457

38. Adewole MB, Sridhar MK, Adeoye GO. Removal of heavy metals from soil polluted with effluents from a paint industry using Helianthus annuus L. and Tithonia diversifolia (Hemsl.) as influenced by fertilizer applications. Bioremediation Journal. 2010 8; 14(4):169-79.

39. Adesodun JK, Atayese MO, Agbaje TA, Osadiaye BA, Mafe OF, Soretire AA. Phytoremediation potentials of sunflowers (Tithonia diversifolia and Helianthus annuus) for metals in soils contaminated with zinc and lead nitrates. Water, Air, and Soil Pollution. 2010; 1(4):195-201. https://doi.org/10.1007/s11270.

40. Ojeniyi SO, Odedina SA, Agbede TM. Soil productivity improving attributes of mexican sunflower (Tithonia diversifolia) and siam weed (Chromolaena odorata). Emirates Journal of Food and Agriculture. 2012; 24(3):243-247. http://www.ejfa.me/index.php/journal/article/view/867.

41. NAGARAJ S, NIZAR-B M. Wild sunflower as a green manure for rice in the mid-country west zone, Tropical Agriculture 1982; (138):69-80.

42. Kolawole OK, Awodun MA, Ojeniyi SO. Soil fertility improvement by Tithonia diversifolia (Hemsl.) A Gray and its effect on Cassava performance and yield. International Journal of Engineering and Science. 2014; 3(8):36-43. http://www.theijes.com/papers/v3-i8/Version-4/F0384036043.pdf

43. Crespo G, Ruiz TE, Álvarez J. Efecto del abono verde de Tithonia (T. diversifolia) en el establecimiento y producción de forraje de P. purpureum vc. Cuba CT-169 y en algunas propiedades del suelo. Revista Cubana de Ciencia Agrícola. 2011; 45(1) 79-82.

44. Jama, B., et al. Tithonia diversifolia as a green manure for soil fertility improvement in western Kenya: a review. Agroforestry systems (2000); 49(2):201-221. https://doi.org/10.1023/A:1006339025728

45. Van Sao, N., Mui, N. T., \& Van Binh, Đ. Biomass production of Tithonia diversifolia (Wild Sunflower), soil improvement on sloping land and use as high protein foliage for feeding goats. Livestock Research for Rural Development. 2010; 22, Article \#151

46. Gachengo CN, Palm CA, Jama B, Othieno C. Tithonia and senna green manures and inorganic fertilizers as phosphorus sources for maize in Western Kenya. Agroforestry systems. 1998; 44(1):21-35. https:// doi.org/10.1023/A:1006123404071

47. Hue NV. Effects of organic acids/anions on P sorption and phytoavailability in soils with different mineralogies. Soil Science. 1991; 152(6):463-471.

48. Cong PT, Merckx R. Improving phosphorus availability in two upland soils of Vietnam using Tithonia diversifolia H. Plant and Soil. 2005; 269(1-2):11-23. https://doi.org/10.1007/s11104-004-1791-1

49. Lasat MM. Phytoextraction of metals from contaminated soil: a review of plant/soil/metal interaction and assessment of pertinent agronomic issues. Journal of Hazardous Substance Research. 1999; 2(1):1-25 https:// doi.org/10.4148/1090-7025.1015

50. Padmavathiamma PK, Li LY. Phytoremediation technology: hyper-accumulation metals in plants. Water, Air, and Soil Pollution. 2007; 184(1-4):105-126. https://doi.org/10.1007/s11270-007-9401-5

51. Ríos-C I, Salazar A. Botón de oro (Tithonia diversifolia (Hemsl.) Gray) una fuente proteica alternativa para el trópico; (Primera parte). Liv. Res. Rural Dev. 1995; 6 Article \#25. 
52. Mejía-Díaz E, Mahecha-Ledesma L, Angulo-Arizala J. Tithonia diversifolia: specie for grazing in silvopastoral systems and methods for estimating consumption. Agronomía Mesoamericana. 2017; 28(1):289-302. http:// revistas.ucr.ac.cr/index.php/agromeso/article/view/22673/27396

53. Rivera JE, Cuartas CA, Naranjo JF, Tafur O, Hurtado EA, Arenas FA, Chará J, Murgueitio E. Efecto de la oferta y el consumo de Tithonia diversifolia en un sistema silvopastoril intensivo (SSPi), en la calidad y productividad de leche bovina en el piedemonte Amazónico colombiano. Liv. Res. Rural Dev. 2015; 27(10):189. http:// www.lrrd.org/lrrd27/10/rive27189.html

54. Rüngeler P, Lyß G, Castro V, Mora G, Pahl HL, Merfort I. Study of three sesquiterpene lactones from Tithonia diversifolia on their anti-inflammatory activity using the transcription factor NF- $\kappa \mathrm{B}$ and enzymes of the arachidonic acid pathway as targets. Planta medica. 1998; 64(7):588-593. DOI: 10.1055/s-2006-957527

55. Mahecha L, Escobar JP, Suárez JF, Restrepo LF. Tithonia diversifolia (hemsl.) Gray (botón de oro) como suplemento forrajero de vacas F1 (Holstein por Cebú). Liv. Res. Rural Dev. 2007; 19(2):1-6. http:// www.lrrd.org/lrrd19/2/mahe19016.htm

56. Lezcano Y, Soca M, Sánchez LM, Ojeda F, Olivera Y, Fontes D, Montejo IL, Santana H. Caracterización cualitativa del contenido de metabolitos secundarios en la fracción comestible de Tithonia diversifolia (Hemsl.) A. Gray. Pastos y Forrajes. 2012; 35(3):283-291.

57. Charmley E. Towards improved silage quality-A review. Canadian Journal of Animal Science. 2001; 81(2):157-168. https://doi.org/10.4141/A00-066

58. Dávila-Hidalgo A, Lepe-Lopez M, Polanco E, Saavedra C, Guerra-Centeno D. Determinación del valor nutricional y evaluación sensorial del ensilado de Sorghum vulgare y Tithonia diversifolia. REDVET. Revista Electrónica de Veterinaria. 2016; 17(10):1-11.

59. Gutiérrez D, Morales A, Elías A, García R, Sarduy L. Composición química y degradabilidad ruminal in situ de la materia seca en ensilajes mixtos Tithonia diversifolia: Pennisetum purpureum vc. Cuba CT-169, inoculados con el producto biológico VITAFERT. Revista Cubana de Ciencia Agrícola. 2014; 48:379-385:

60. Pinto R, Hernández D, Guevara F, gómez H, Medina F, Hernández A et al. Preferencia de ovinos por el ensilaje de Pennisetum purpureum mezclado con arbóreas forrajeras tropicales. Liv. Res. Rural Dev. 2010; 22, Article \#106. http://www.lrrd.org/lrrd22/6/pint22106.htm

61. Corporación colombiana de investigación agropecuaria - CORPOICA 2013. Sistema de Toma de Decisión para la selección de especies Forrajeras STDF ficha Tithonia diversifolia. Disponible en: http:// www.corpoica.org.co:8086/NetCorpoicaMVC/STDF/Content/fichas/pdf/Ficha_102.pdf

Los autores permiten a RECIA reimprimir el material publicado en él. En caso de que un autor quiera traducir o usar una publicación parcial o completa de nuestro Diario, el autor debe obtener un permiso por escrito del editor de la revista.

CC BY 